\title{
Food-Induced Duodenal Obstruction Successfully Reopened by Endoscopic Treatment
}

\author{
Satoshi Masuda ${ }^{1}$, Taiki Aoyama ${ }^{1}$, Akira Fukumoto ${ }^{1}$, Shinji Nagata ${ }^{1}$ \\ 1. Gastroenterology, Hiroshima City Asa Citizens Hospital, Hiroshima, JPN
}

Corresponding author: Taiki Aoyama, t-aoyama@asa-hosp.city.hiroshima.jp

\section{Abstract}

Duodenal obstruction is a rare event that is unlikely to be treated endoscopically. Herein, we describe the case of a 75-year-old woman who presented with vomiting and was diagnosed with food-induced duodenal obstruction. Impacted food was fragmented and removed by double-balloon enteroscopy, and the duodenal tract was reopened without any adverse events. Follow-up capsule endoscopy was performed one month after treatment to determine the obstruction etiology and it revealed a remarkably delayed passage of the capsule through the duodenum and excessive amounts of floating food residue in the third portion of the duodenum. Obstruction recurrence was not observed six months after endoscopic treatment. In conclusion, in our case, endoscopic treatment of duodenal obstruction prevented the unnecessary performance of surgery, suggesting its clinical utility for this condition.

Review began 11/26/2020 Review ended 12/14/2020 Published 12/19/2020

\section{(c) Copyright 2020}

Masuda et al. This is an open access article distributed under the terms of the Creative Commons Attribution License CC-BY 4.0., which permits unrestricted use, distribution, and reproduction in any medium, provided the original author and source are credited.
Categories: Emergency Medicine, Internal Medicine, Gastroenterology

Keywords: double-balloon enteroscopy, duodenum, endoscopic treatment, food, ileus

\section{Introduction}

Management of an acquired duodenal obstruction is challenging because of the limited treatment options. Owing to the anatomical complexity of the area, even minimally invasive surgical procedures, such as pancreas-sparing or segmental resection of the duodenum, are associated with an $11.8 \%-38 \%$ rate of adverse events, including duodenal leak, fistulas and hemorrhage [1-3]. Transluminal endoscopic treatment could be a viable option as endoscopic treatment is already an important alternative to surgery for cases of hemostasis and removal of a foreign body from the small bowel [4-6].

In this report, we describe a case of food-induced mechanical obstruction in the third portion of the duodenum. Endoscopic treatment was selected instead of surgery to reopen the obstruction.

\section{Case Presentation}

A 75-year-old woman with no history of abdominal surgeries was admitted to our hospital with a chief complaint of recent-onset vomiting lasting two days. The patient had a history of type 2 diabetes mellitus and reported a history of chronic abdominal discomfort. Abdominal computed tomography (CT) revealed a mechanical obstruction with a high-density mass in the third portion of the duodenum (Figure 1).

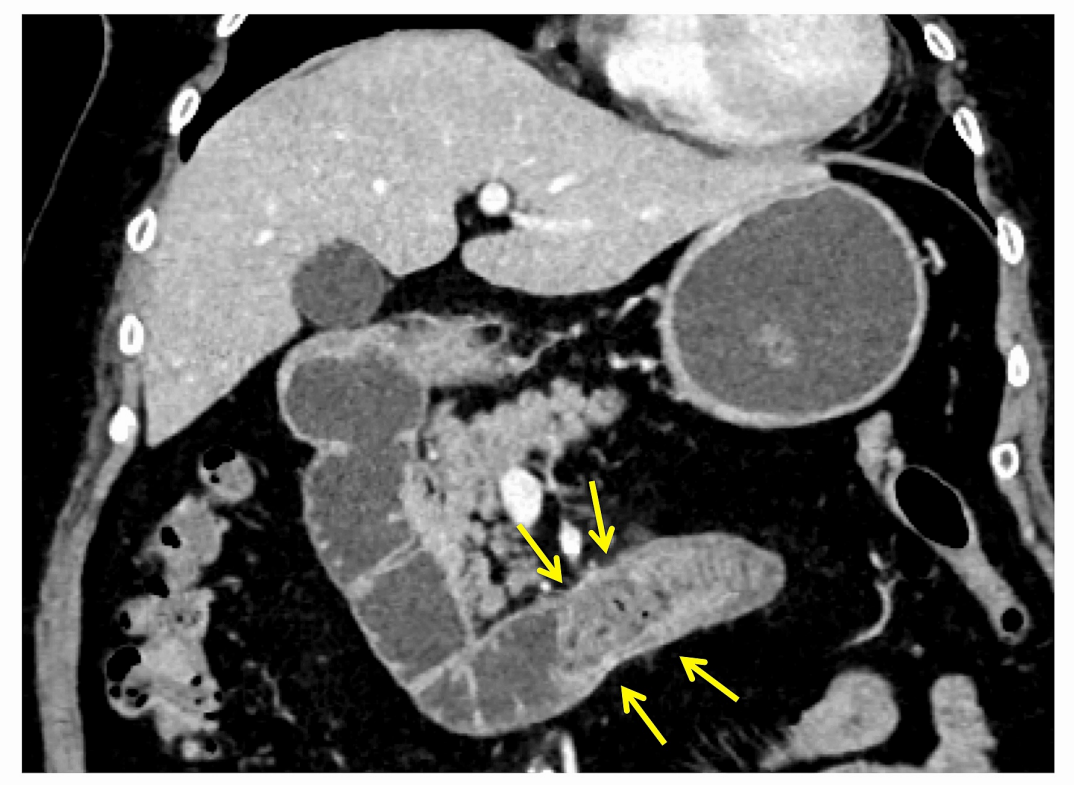




\section{Cureus}

\section{FIGURE 1: Abdominal computed tomography at admission}

Bowel obstruction is seen in the third portion of the duodenum (yellow arrows).

After draining the pooled liquid in the stomach using a nasogastric tube, double-balloon enteroscopy (DBE) was used to confirm the etiology because the mass was not located within the reach of the

esophagogastroduodenoscope. A food complex with a jelly-like appearance, which was not suspected to be a neoplasm or a gallstone, was impacted at the site (Figure $2 a$ ). The food mass was tightly fixed, immobile, and was too fragile to be grasped using forceps. Instead of surgery, we attempted an endoscopic removal of the mass using the SnareMaster (Olympus, Tokyo, Japan) (Figure $2 b$ ). We performed fragmentation without energization for 1 hour. The food complex was elaborately fragmented into small pieces to avoid impacting the distal small bowel. Thereafter, the duodenal tract was reopened, demonstrating the success of endoscopic disimpaction in this case (Figure 2c). The scope was advanced to the jejunum beyond the Treitz' ligament without any resistance. In addition to this, no stricture was identified, which was verified using the radiological contrast test during DBE. Ulcers and surrounding mucosal edema observed at the site were considered to have developed from the ischemic injury caused by the pressure of the impacted food complex, which had the same etiology as that of solitary rectal ulcers [7]. Biopsy specimens obtained at the site showed no specific findings, and Crohn's disease, eosinophilic enteropathy, and amyloid deposits were not suspected. The peristalsis of the area appeared relatively weak . An abdominal CT scan obtained the next day showed no evidence of perforation, and the ileus had disappeared (Figure 3). The patient was discharged 7 days after treatment without any adverse events.
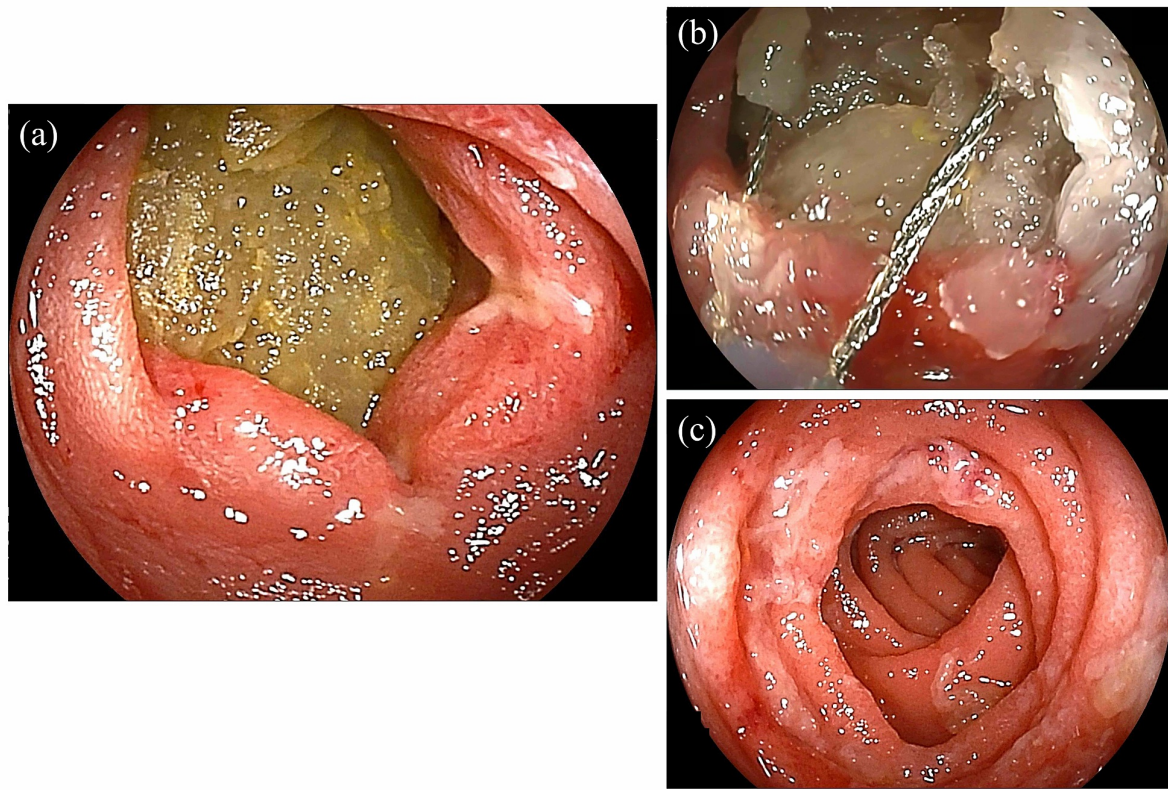

FIGURE 2: Endoscopic finding of the duodenum

(a) Food complex impacted in the lumen

(b) Removal of the surface of the mass using an endoscopic snare

(c) Re-opening of the duodenal tract 


\section{Cureus}

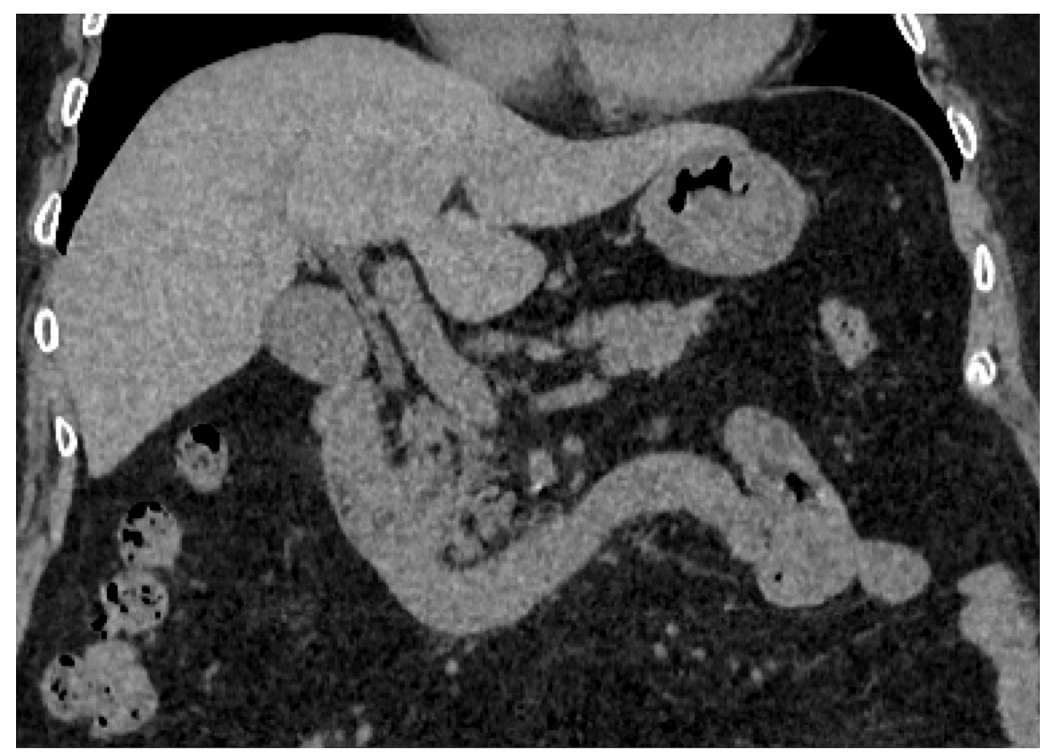

FIGURE 3: The abdominal computed tomography findings obtained the next day

It shows no evidence of perforation, and the ileus had disappeared.

Capsule endoscopy (CE) examination was performed one month after discharge to assess the entire small bowel. No abnormalities were noted in the jejunum or ileum. A delayed passage time of 120 minutes within the duodenum accounted for $39 \%$ of the total small bowel exploration time, and food residue was observed floating in the third portion of the duodenum, suggesting that the abnormal motility of the duodenum caused food clustering, resulting in a bowel obstruction (Figure 4). The patient had not taken non-steroidal anti-inflammatory drugs or any medication that could decrease the motility of the duodenum. Her diabetes was well controlled, as indicated by a hemoglobin A1c of 6.6\%. Moreover, hypothyroidism was not observed as her thyroid-stimulating hormone (normal range, $0.34-4.0 \mu \mathrm{IU} / \mathrm{mL}$ ) and free thyroxine 4 (normal range, $0.8-1.7 \mathrm{ng} / \mathrm{mL}$ ) levels were $2.76 \mu \mathrm{IU} / \mathrm{mL}$ and $1.03 \mathrm{ng} / \mathrm{dL}$ respectively. She had no history of alcohol consumption. Recurrence of an obstruction was not seen after 6 months owing to the administration of the medication that increased gastroduodenal motility after the endoscopic treatment. Follow-up DBE confirmed no residual abnormality at the site (Figure 5). 


\section{Cureus}

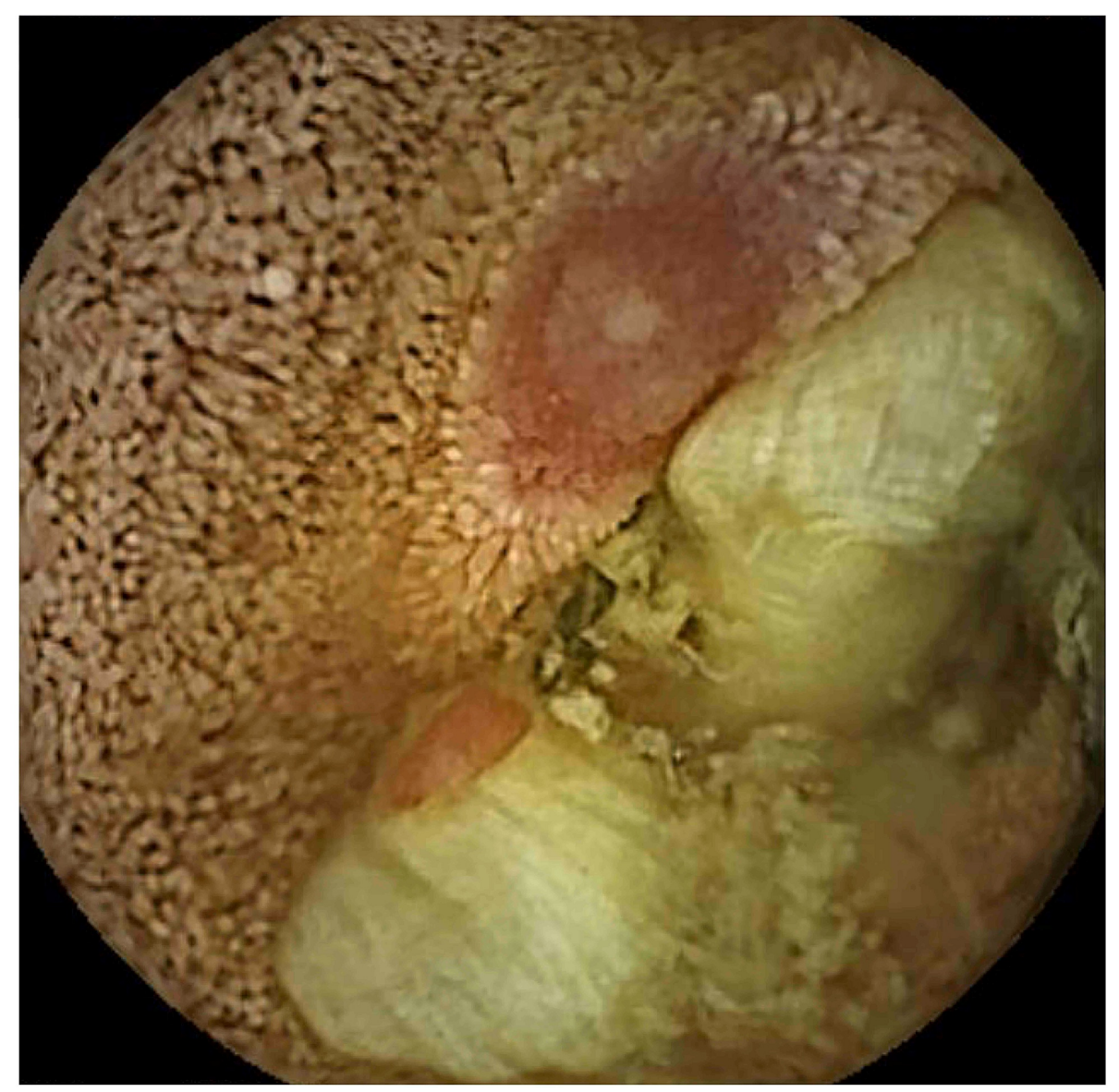

FIGURE 4: Capsule endoscopic examination

Delayed passage of a food residue in the third portion of the duodenum.

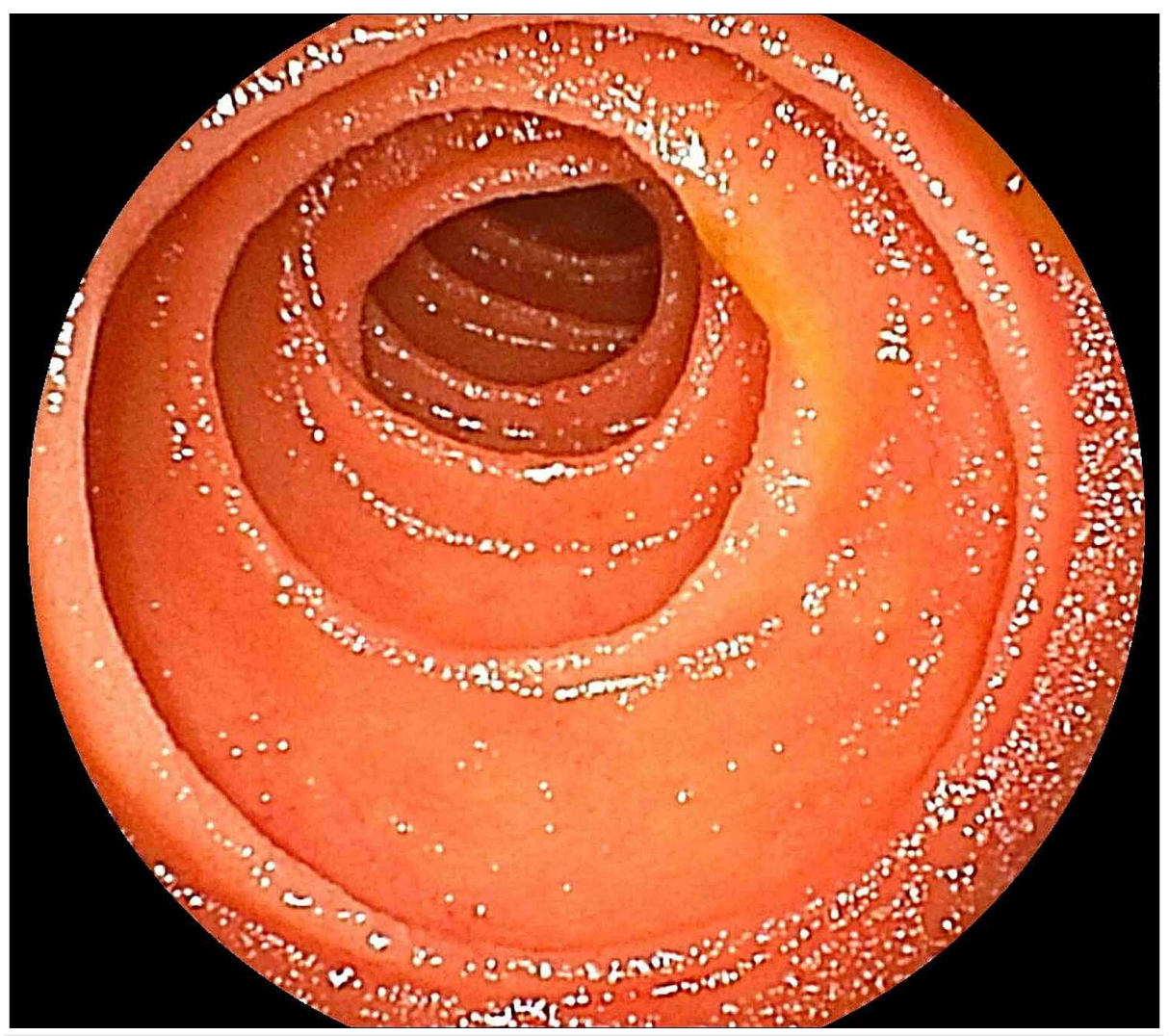


FIGURE 5: Double-balloon enteroscopic finding 6 months after treatment

Neither ulcer nor stricture is observed at the treated site.

\section{Discussion}

While cases of food-derived ileus have been reported, it remains a rare condition. Fourteen cases of small bowel ileus induced by rice cakes have been reported, all of which were resolved after conservative treatment [8]. A case of jejunal ileus due to an impaction of shiitake mushrooms was reported, which was treated endoscopically using a snare to fragment the impaction [9], similar to our case. Other ileus cases induced by foreign bodies, such as gallstones, phytobezoars, and enteroliths, have also been reported [10-12]. Most of these cases required surgical treatment for disimpaction. When retrospectively reviewing our case, conservative treatment was not likely to release the obstruction due to the tight impaction of the food complex. Due of the anatomical complexity of the duodenum and a lack of evidence for neoplasm in this case, a surgical procedure would have been excessive. Therefore, endoscopic treatment was considered a reasonable option.

The etiology of the duodenal obstruction in this case is still obscure. Instead of manometry or magnetic resonance enterography, CE was used to determine the etiology in this case after confirming the absence of a small bowel stricture using CT (e.g., we confirmed a lack of both intestinal narrowing with distension at the oral side and extensive bowel wall thickness [13]). Not only did CE reveal the food residue retention, but also the abnormal motility of the duodenum. Although CE has the same diagnostic ability for small bowel lesions as DBE [14], CE can also obtain information about the physiological activity of the small bowel. In this case, a remarkably delayed transit time through the duodenum caused food clustering at the site, which resulted in an obstruction. Functional dyspepsia (FD) and diabetic neuropathy could be etiologies of the duodenal dysmotility in our case.

Changes in the number of intestinal cells of Cajal and myenteric neurons due to impaired mucosal integrity and low baseline impedance in the duodenum which result in abnormal gastroduodenal motility, have been reported in patients with $\mathrm{FD}[15,16]$. Alternatively, a high-fat diet and microbial alteration are thought to cause enteric diabetic neuropathy and intestinal dysmotility based on the reduction of nitrergic myenteric neurons per ganglion in the duodenum [17]. The median time of retention in the duodenum was reported to be longer in diabetic patients than in healthy volunteers (12.7 versus 8.1 minutes) [18].

\section{Conclusions}

This case demonstrated that endoscopic examination may aid in the treatment of ileus, with more favorable outcomes than conservative therapy. The use of DBE could help make a decision for optimal treatment in cases of ileus. Considering the anatomical complexity of the duodenum, transluminal endoscopic treatment could be a viable option to prevent the unnecessary performance of surgery. Moreover, after resolving the ileus status, CE can be clinically useful in clarifying the etiology as it provides information regarding duodenal motility as well as intra-luminal abnormality.

\section{Additional Information \\ Disclosures}

Human subjects: Consent was obtained by all participants in this study. Conflicts of interest: In compliance with the ICMJE uniform disclosure form, all authors declare the following: Payment/services info: All authors have declared that no financial support was received from any organization for the submitted work. Financial relationships: All authors have declared that they have no financial relationships at present or within the previous three years with any organizations that might have an interest in the submitted work. Other relationships: All authors have declared that there are no other relationships or activities that could appear to have influenced the submitted work.

\section{References}

1. Zhou B, Zhang M, Wu J, Yan S, Zhou J, Zheng S: Pancreaticoduodenectomy versus local resection in the treatment of gastrointestinal stromal tumors of the duodenum. World J Surg Oncol. 2013, 11:1-6. 10.1186/1477-7819-11-196

2. Garcia-Molina FJ, Mateo-Vallejo F, Franco-Osorio Jde D, Esteban-Ramos JL, Rivero-Henandez I: Surgical approach for tumours of the third and fourth part of the duodenum. Distal pancreas-sparing duodenectomy. Int J Surg. 2015, 18:143-8. 10.1016/j.ijsu.2015.04.051

3. Spalding DRC, Isla AM, Thompson JN, Williamson RCN: Pancreas-sparing distal duodenectomy for infrapapillary neoplasms. Ann R Coll Surg Engl. 2007, 89:130-5. 10.1308/003588407X155815

4. Aoyama T, Fukumoto A, Mukai S, Ueda H, Kimura S, Nagata S: Successful endoscopic treatment of an actively bleeding jejunal dieulafoy's lesion. Intern Med. 2016, 55:1739-41. 10.2169/internalmedicine.55.6381 
5. Shishido T, Oka S, Tanaka S, et al.: Removal of a sewing needle penetrating the wall of the third portion of the duodenum by double-balloon endoscopy. Clin J Gastroenterol. 2012, 5:79-81. 10.1007/s12328-011-02741

6. Aoyama T, Fukumoto A, Shigita K, Asayama N, Mukai M, Nagata S: Successful endoscopic sclerotherapy using polidocanol for small bowel hemangioma. Intern Med. 2020, 59:1727-30.

10.2169/internalmedicine.4327-19

7. Ignjatovic A, Saunders BP, Harbin L, Clark S: Solitary 'rectal' ulcer syndrome in the sigmoid colon. Colorectal Dis. 2010, 12:1163-4.

8. Miura T, Kimura N, Nakamura J, et al.: Rice cake ileus - a rare and ethnic but important disease status in east-southern Asia. Intern Med. 2011, 50:2737-9. 10.2169/internalmedicine.50.6152

9. Shozushima M, Ohata K, Nonaka K, Matsuhashi N: Shiitake mushroom-induced ileus managed using double-balloon enteroscopy. Endoscopy. 2013, 45:437. 10.1055/s-0033-1359138

10. Steenvoorde P, Schaardenburgh P, Viersma JH: Enterolith ileus as a complication of jejunal diverticulosis: two case reports and a review of the literature. Dig Surg. 2003, 20:57-60. 10.1159/000068852

11. Gok AFK, Sonmez RE, Kantarci TR, Bayraktar A, Emiroğlu S, İlhan M, Güloğlu R: Discussing treatment strategies for acute mechanical intestinal obstruction caused by phytobezoar: a single-center retrospective study. Ulus Travma Acil Cerrahi Derg. 2019, 25:503-9. 10.14744/tjtes.2019.24557

12. Ploneda-Valencia CF, Gallo-Morales M, Rinchon C, et al.: Gallstone ileus: an overview of the literature . Rev Gastroenterol Mex. 2017, 82:248-54. 10.1016/j.rgmx.2016.07.006

13. Nakano M, Oka S, Tanaka S, et al.: Clinical usefulness of classification by transabdominal ultrasonography for detection of small-bowel stricture. Scand J Gastroenterol. 2013, 48:1041-7. 10.3109/00365521.2013.822546

14. Shishido T, Oka S, Tanaka S, et al.: Diagnostic yield of capsule endoscopy vs. double-balloon endoscopy for patients who have undergone total enteroscopy with obscure gastrointestinal bleeding. Hepatogastroenterology. 2012, 59:955-9. 10.5754/hge12242

15. Nakagawa K, Hara K, Fikree A, et al.: Patients with dyspepsia have impaired mucosal integrity both in the duodenum and jejunum: in vivo assessment of small bowel mucosal integrity using baseline impedance. J Gastroenterol. 2020, 55:273-80. 10.1007/s00535-019-01614-5

16. Tack J, Van den Houte K, Carbone F: Gastroduodenal motility disorders. Curr Opin Gastroenterol. 2018, 34:428-35. 10.1097/MOG.0000000000000473

17. Nyavor Y, Brands CR, May G, et al.: High-fat diet-induced alterations to gut microbiota and gut-derived lipoteichoic acid contributes to the development of enteric neuropathy. Neurogastroenterol Motil. 2020, 32:13838. 10.1111/nmo.13838

18. Jacob VYP, Felber J, Muller N, Kloos C, Müller UA, Stallmach A: The high-resolution three-dimensional magnetic detector system 3D-magna accurately measures gastric and small bowel motility in people with type 2 diabetes with neuropathy. Exp Clin Endocrinol Diabetes. 2020, Online of ahead of print: 10.1055/a1163-7230 